\title{
EXISTENCE OF PERIODIC TRAVELING WAVE SOLUTIONS TO THE GENERALIZED FORCED BOUSSINESQ EQUATION
}

\author{
KENNETH L. JONES and YUNKAI CHEN
}

\author{
(Received 3 September 1998)
}

\begin{abstract}
The generalized forced Boussinesq equation, $u_{t t}-u_{x x}+[f(u)]_{x x}+u_{x x x x}=$ $h_{0}$, and its periodic traveling wave solutions are considered. Using the transform $z=$ $x-\omega t$, the equation is converted to a nonlinear ordinary differential equation with periodic boundary conditions. An equivalent relation between the ordinary differential equation and a Hammerstein type integral equation is then established by using the Green's function method. This integral equation generates compact operators in a Banach space of realvalued continuous periodic functions with a given period $2 T$. The Schauder's fixed point theorem is then used to prove the existence of solutions to the integral equation. Therefore, the existence of nonconstant periodic traveling wave solutions to the generalized forced Boussinesq equation is established.
\end{abstract}

Keywords and phrases. Existence theorem, periodic traveling wave solutions, generalized forced Boussinesq equation.

1991 Mathematics Subject Classification. 35Q53, 35B10, 35Q20.

1. Introduction. In the 1870's, Boussinesq derived some model equations for the unidirectional propagation of small amplitude long waves on the surface of water [2]. These equations possess special traveling wave solutions called solitary waves. Boussinesq's theory was the first to give a satisfactory and scientific explanation of the phenomenon of solitary waves discovered by Scott Russell [8].

The original equation obtained by Boussinesq is not the only mathematical model for small amplitude, planar, and undirectional long waves on the surface of shallow water. Different choices of the independent variables and the possibilty of modifying lower order terms by the use of the leading order relationships can lead to a whole range of equations [1]. One of them is the well-known Korteweg-de Vries equation (referred to $\mathrm{KdV}$ equation henceforth). In a recent paper, Schneider proved that under certain conditions, solutions of the Boussinesq equation can be split up into two approximate solutions of $\mathrm{KdV}$ equation [7].

Twenty years ago, in an impressive survey on the KdV equation, Miura listed seven open problems on that equation [6]. The seventh open problem is on the forced KdV equation. In 1995, Shen derived the 1-dimensional stationary forced KdV equation of the form $\lambda u_{t}+\alpha u u_{x}+\beta u_{x x x}=h_{x}$ for the long nonlinear water waves flowing over long bumps, and proved the existence of positive solitary wave solutions to the equation with boundary conditions $u( \pm \infty)=u^{\prime}( \pm \infty)=0$ [9]. In a recent paper [3], Chen proved the existence of traveling wave solutions to the generalized forced KadomtsevPetviashvili equation which is a 2-dimensional generalization to the equation obtained 
by Shen.

In this paper, we consider an equation of the form

$$
u_{t t}-u_{x x}+[f(u)]_{x x}+u_{x x x x}=h_{0},
$$

where $u=u(t, x)$ and $f(u)$ is a $C^{2}$ function in its argument. This equation is also called the generalized forced Boussinesq equation.

We shall prove an existence theorem of periodic traveling wave solutions to this equation following the idea of Liu and Pao [5], and Chen and He [4].

The plan of this paper is as follows. In Section 2, the generalized forced Boussinesq equation is transformed to an ordinary differential equation with periodic boundary conditions. We then apply the Green's function method to derive a nonlinear integral equation equivalent to the ordinary differential equation. In Section 3, an existence theorem of solutions to the integral equation is proved. Therefore, the main result, the existence of periodic traveling wave solutions to the generalized forced Boussinesq equation is established. Furthermore, we note that the periodic traveling wave solutions are infinitely differentiable.

2. Formulation of the problem. We start from the generalized forced Boussinesq equation of the form

$$
u_{t t}-u_{x x}+[f(u)]_{x x}+u_{x x x x}=h_{0},
$$

where the function $f$ is $C^{2}$ in its argument and $h_{0}$ is a nonconstant function of $t$ and $x$. We are interested in the periodic traveling wave solutions of the form $u(x, t)=$ $U(z)=U(x-\omega t)$, where $\omega>0$ is the wave speed and $z=x-\omega t$ is the characteristic variable. In this paper, we only consider the case that $h_{0}(x, t)=h(z)$ is a $2 T$-periodic continuous function of $z$, where $T$ is a preassigned positive number. Substituting the $U(z)$ into equation (2.1) then leads to the fourth order nonlinear ordinary differential equation

$$
U^{(4)}(z)=C U^{\prime \prime}(z)-[f(U(z))] "+h(z),
$$

where $C=\left(1-\omega^{2}\right)$. To obtain nonzero, nonconstant, periodic solutions of this equation, we impose the following boundary conditions

$$
\begin{gathered}
U^{(n)}(0)=U^{(n)}(2 T), \quad n=0,1,2,3, \\
\int_{0}^{2 T} U(z) d z=0 .
\end{gathered}
$$

It is obvious that any solution $U(z)$ of the boundary value problem consisting of equations (2.2), (2.3), and (2.4) can be extended to a $2 T$-periodic traveling wave solution to the original Boussinesq equation (2.1).

Integrating both sides of equation (2.2) with respect to $z$ twice and using equations (2.3) and (2.4) yield

$$
\begin{aligned}
U^{\prime \prime}(z)-C U(z) & =E-[f(U(z))-H(z)], \\
U^{(n)}(0) & =U^{(n)}(2 T), \quad n=0,1,
\end{aligned}
$$

where $E=\int_{0}^{2 T}[f(U(z))-H(z)] d z / 2 T$, and $H(z)$ is a $2 T$-periodicfunction of $z$ such 
that $H^{\prime \prime}(z)=h(z)$. Conversely, integrating both sides of equation (2.5) from 0 to $2 T$ and using equation (2.6) will give us equation (2.4), and direct differentiations of equation (2.5) will give us equations (2.2) and (2.3). Therefore, we have proved the following theorem by noting from equation (2.5) that $U \in C^{2}[0,2 T]$ implies $U \in C^{4}[0,2 T]$ because $f$ is a $C^{2}$ function of its argument.

THEOREM 1. Suppose $C \neq 0$, a function $U(z)$ is a solution to the boundary value problem equations (2.2), (2.3), and (2.4) if and only if it is a solution to the boundary value problem consisting of equations (2.5) and (2.6).

From now on we denote the function $f(U(z))-H(z)$ on the right hand side of equation (2.5) by $F(U(z))$ and only consider the two cases:

(1) $C>0$,

(2) $C<0$,

but $-C \neq(k \pi / T)^{2}$ with $k$ being any integer. Treating the right hand side of equation (2.5) as a forcing term and using the Green's function method [5, 11, 10], the boundary value problem equations (2.5) and (2.6) can be converted to a nonlinear integral equation of the form

$$
U(z)=\int_{0}^{2 T} K_{i}(z, s) F(U(s)) d s \quad \forall z \in[0,2 T],
$$

where the kernels $K_{i}, i=1,2$, are defined as follows:

(1) When $C>0$, we denote $\lambda_{1}=\sqrt{C}$, then

$$
K_{1}(z, s)=\frac{\cosh \lambda_{1}(T-|z-s|)}{2 \lambda_{1} \sinh \lambda_{1} T}-\frac{1}{2 \lambda_{1}^{2} T} \quad \forall z, s \in[0,2 T] .
$$

(2) When $C<0$ but $-C \neq(k \pi / T)^{2}$ with $k$ being any integer, let $\lambda_{2}=\sqrt{-C}$, then

$$
K_{2}(z, s)=\frac{\cos \lambda_{2}(T-|z-s|)}{2 \lambda_{2} \sin \lambda_{2} T}-\frac{1}{2 \lambda_{2}^{2} T} \quad \forall z, s \in[0,2 T] .
$$

LEMMA 1. The kernels $K_{1}$ and $K_{2}$ have the following properties:

$$
\begin{gathered}
K_{i}(0, s)=K_{i}(2 T, s) \quad \forall s \in[0,2 T], i=1,2, \\
K_{i}(z, 2 T-s)=K_{i}(2 T-z, s) \quad \forall z, s \in[0,2 T], i=1,2, \\
\int_{0}^{2 T} K_{i}(z, s) d s=0 \quad \forall z \in[0,2 T], i=1,2 .
\end{gathered}
$$

Proof. Straightforward computations from the definitions of the $K_{1}(z, s)$ and $K_{2}(z, s)$ given in equations (2.8) and (2.9).

THEOREM 2. A function $U(z)$ is a solution of the boundary value problem consisting of equations (2.5) and (2.6) if and only if it is a solution of the integral equation (2.7).

Proof. The if part can be proved by direct differentiations of equation (2.7) and the only if part is based on the Green's function method by treating the right hand side of equation (2.5) as a nonhomogeneous term. 
3. Existence theorem. It is seen from Theorems 1 and 2 that $U(z)$ is a solution to the integral equation (2.7) if and only if it is a solution to the boundary value problem consisting of equations (2.2), (2.3), and (2.4). Therefore, to show the existence of $2 T$ periodic traveling wave solutions to the generalized forced Boussinesq equation it is sufficient to show that solutions to equation (2.7) exist.

We define $C_{2 T}$ as a collection of real-valued continuous functions, $v(z)$, on $[0,2 T]$ such that $v(0)=v(2 T)$. Equip $C_{2 T}$ with the sup norm $\|\cdot\|$ as $\|v\|=\sup _{0 \leq z \leq 2 T}|v(z)|$, for each $v \in C_{2 T},\left(C_{2 T},\|\cdot\|\right)$ then becomes a Banach space.

We now define the operators $\mathscr{A}_{i}, i=1,2$, on $C_{2 T}$ as

$$
\mathscr{A}_{i} v(z)=\int_{0}^{2 T} K_{i}(z, s) F(v(s)) d s \quad \forall v \in C_{2 T},
$$

where the kernels $K_{i}, i=1,2$, are defined in equations (2.8), (2.9), and

$$
F(v(s))=f(v(s))-H(s) .
$$

Notice that the operator $\mathscr{A}_{i}$ depends on $T$ and $\lambda_{i}, i=1,2$.

We shall show that there exists an $r>0$ such that $\left\|\mathscr{A}_{i} v\right\| \leq r$ for any nontrivial function $v \in B(0, r) \subseteq C_{2 T}, i=1,2$. This implies that the equation $\mathscr{A}_{i} v=v$ has at least one solution in $B(0, r)$. And hence, the existence of solutions to equation (2.7) is therefore established. This, in turn, leads to the existence of $2 T$-periodic traveling wave solution $U(z)$ to the generalized forced Boussinesq equation.

A consequence of Lemma 1 can be stated now.

LEMMA 2. Let $v$ be an element of $C_{2 T}$. If $v(z)=v(2 T-z)$ for $z \in[0,2 T]$, then $\mathscr{A}_{i} v(z)=\mathscr{A}_{i} v(2 T-z), i=1,2$.

We define $B(0, r)$ as a bounded ball in $C_{2 T}$ with $r>0$, then there exists an $M>0$ such that $M=\sup [\|F(v)\|: v \in B(0, r)]$. We are now ready to prove the following theorem:

THEOREM 3. $\mathscr{A}_{i}: C_{2 T} \rightarrow C_{2 T}, i=1,2$, is a compact operator. If $2 M / \lambda_{i}^{2} \leq r, i=1,2$, then $\mathscr{A}_{i}, i=1,2$ maps $B(0, r)$ into itself, and hence, the integral equation (2.7) has at least one solution in $B(0, r)$.

Proof. First we show that $\mathscr{A}_{i}: C_{2 T} \rightarrow C_{2 T}, i=1,2$. Since it is obvious from Lemma 1 that $\mathscr{A}_{i} v(0)=\mathscr{A} v(2 T)$ for each $v \in C_{2 T}, i=1,2$, it suffices to show that $\mathscr{A}_{i} v, i=1,2$, is continuous on $[0,2 T]$.

Let $v$ be an element in $C_{2 T}$, we have

$$
\begin{aligned}
\frac{d A_{1} v(z)}{d z}= & \frac{-1}{2 \sinh \lambda_{1} T} \int_{0}^{z} \sinh \lambda_{1}(T-z+s) F(v(s)) d s \\
& +\frac{1}{2 \sinh \lambda_{1} T} \int_{z}^{2 T} \sinh \lambda_{1}(T+z-s) F(v(s)) d s \\
\frac{d A_{2} v(z)}{d z}= & \frac{1}{2 \sin \lambda_{2} T} \int_{0}^{z} \sin \lambda_{2}(T-z+s) F(v(s)) d s \\
& +\frac{-1}{2 \sin \lambda_{2} T} \int_{z}^{2 T} \sin \lambda_{2}(T+z-s) F(v(s)) d s
\end{aligned}
$$


The existence of $d \mathscr{A}_{1} v / d z$ and $d \mathscr{A}_{2} v / d z$ implies that both $\mathscr{A}_{1} v$ and $\mathscr{A}_{2} v$ are continuous on $[0,2 T]$, and hence, we have proved $\mathscr{A}_{i}: C_{2 T} \rightarrow C_{2 T}, i=1,2$.

Let $\mathbf{S}$ be any bounded subset of $C_{2 T}$, i.e., there exists an $L_{0}>0$ such that $\|v\| \leq L_{0}$ for all $v \in \mathbf{S}$. Then since $f$ is $C^{2}$ in its argument, there exists an $M_{0}>0$ such that

$$
\|F(v)\|=\sup _{0 \leq z \leq 2 T}|F(v(z))| \leq \sup _{-L_{0} \leq \omega \leq L_{0}}|F(\omega)| \leq M_{0} \quad \forall v \in \mathbf{S} .
$$

Since $\sin \lambda_{2} T \neq 0$ and $\max _{0 \leq z \leq 2 T} \int_{0}^{2 T}\left|K_{i}(z, s)\right| d s \leq 2 / \lambda_{i}^{2}, i=1,2$ [10], we can obtain the following results from equations (3.1), (3.2), and (3.3)

$$
\begin{gathered}
\left\|\mathscr{A}_{i} v\right\| \leq \frac{2 M_{0}}{\lambda_{i}^{2}} \quad \forall v \in \mathbf{S}, i=1,2, \\
\left\|\frac{d \mathscr{A}_{i} v}{d z}\right\| \leq \frac{T M_{0}}{T_{i}} \quad \forall v \in \mathbf{S}, i=1,2,
\end{gathered}
$$

where $\tau_{1}=1$ and $\tau_{2}=\left|\sin \lambda_{2} T\right|$. Thus, $\mathscr{A}_{i} \mathbf{S}, i=1,2$, is uniformly bounded and equicontinuous, and by the Ascoli-Arzela theorem both $\mathscr{A}_{1}$ and $\mathscr{A}_{2}$ are compact operators from $C_{2 T}$ into $C_{2 T}$.

To show that $\mathscr{A}_{i}, i=1,2$, has a fixed point in $B(0, r)$ when $2 M / \lambda_{i}^{2} \leq r, i=1,2$, we write

$$
\begin{aligned}
\left|\mathscr{A}_{i} v(z)\right| & =\left|\int_{0}^{2 T} K_{i}(z, s) F(v(s)) d s\right| \\
& \leq \int_{0}^{2 T}\left|K_{i}(z, s)\right|\|F(v(s))\| d s \\
& \leq \frac{2 M}{\lambda_{1}^{2}} \leq r, \quad \forall v \in B(0, r) .
\end{aligned}
$$

This implies that $\left\|\mathscr{A}_{i} v\right\| \leq r$ for all $v \in B(0, r), i=1,2$. And hence, $\mathscr{A}_{i}, i=1,2$, maps $B(0, r)$ into itself. By the Shauder's fixed point theorem we have proved that $\mathscr{A}_{i}$, $i=1,2$ has a fixed point in $B(0, r)$. This means that the equation $\mathscr{A}_{i} v=v, i=1,2$, has at least a solution $v(z)$ which is continuous on $[0,2 T]$. This, in turn, implies that equation (2.7) has a solution for each case of $C>0$ and $C<0$ with $-C \neq(k \pi / T)^{2}$.

It is worth nothing that as long as $\int_{0}^{2 T} K_{i}(z, s) H(s) d s \neq 0, i=1,2$, by Theorem 3 , we see that the equation $\mathscr{A}_{i} v=v, i=1,2$, has at least one nonconstant solution $v(z)$ in $B(0, r)$. This solution $v(z)$ is infinitely differentiable on $(0,2 T)$ since $\mathscr{A}_{i} v$ is differentiable on $(0,2 T)$. The extension of the $v(z)$ to a $2 T$-periodic function $V(z)$ provides an infinitely differentiable nonconstant $2 T$-periodic traveling wave solution to the generalized forced Boussinesq equation.

\section{REFERENCES}

[1] J. L. Bona and R. Smith, A model for the two-way propagation of water waves in a channel, Math. Proc. Cambridge Philos. Soc. 79 (1976), no. 1, 167-182. MR 5216254. Zbl 332.76007 .

[2] J. Boussinesq, Theorie de l'intumescence liquide appelee onde solitaire ou de translation se propageant dans un canal rectangulare, Compte Rendus Acad Sci. 72 (1871), 755-759. 
[3] Y. Chen, On existence theorems of periodic traveling wave solution to the generalized forced Kadomtsev-Petviashvili equation, Arch. Mech. (Arch. Mech. Stos.) 48 (1996), no. 5, 925-931. MR 97k:76014. Zbl 872.35094.

[4] Yunkai Chen and Xiaogui He, Nonexistence of small periodic traveling wave solutions to the power Kadomtsev-Petviashvili equation, Differ. Equ. Dyn. Syst 5 (1997), no. 1, 43-50. Zbl 891.34021.

[5] Bao-Ping Liu and C. V. Pao, On periodic traveling wave solutions of Boussinesq equation, Quart. Appl. Math. 42 (1984), no. 3, 311-319. MR 86i:35128. Zbl 598.76019.

[6] R. M. Miura, The Korteweg-de Vries equation: a survey of results, SIAM Rev. 18 (1976), no. 3, 412-459. MR 53 8689. Zbl 333.35021.

[7] G. Scheider, The long wave limit for a Boussinesq equation, SIAM J. Appl. Math. 58 (1998), no. 4, 1237-1245. Zbl 980.38665.

[8] J. Scott Russell, Report on waves, 14th Meeting of the British Association Report, 1844, York, pp. 311-390.

[9] S. S. P. Shen, On the accuracy of the stationary forced Korteweg-de Vries equation as a model equation for flows over a bump, Quart. Appl. Math. 53 (1995), no. 4, 701-719. MR 96i:76019. Zbl 859.76008.

[10] E. Soewono, A remark on a paper by Liu and Pao on the existence of periodic travelling wave solution to the KdV equation, Applicable Anal. 25 (1987), no. 4, 293-299. MR 89d:35171. Zbl 609.35079.

[11] I. Stakgold, Green's functions and boundary value problems, Pure and Applied Mathematics, John Wiley \& Sons, New York, 1979. MR 80k:35002. Zbl 421.34027.

Jones and Chen: Department of Mathematics and Computer Science, Fayetteville StATE UNIVERsity, FAYETTEVIlle, NorTh CAROlin 28301-4298, USA 


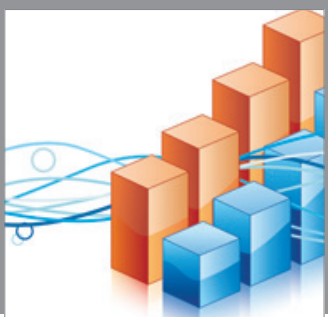

Advances in

Operations Research

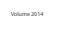

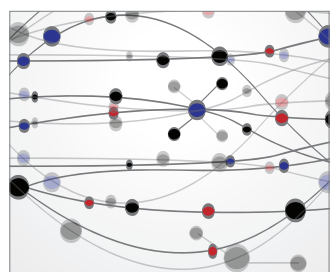

\section{The Scientific} World Journal
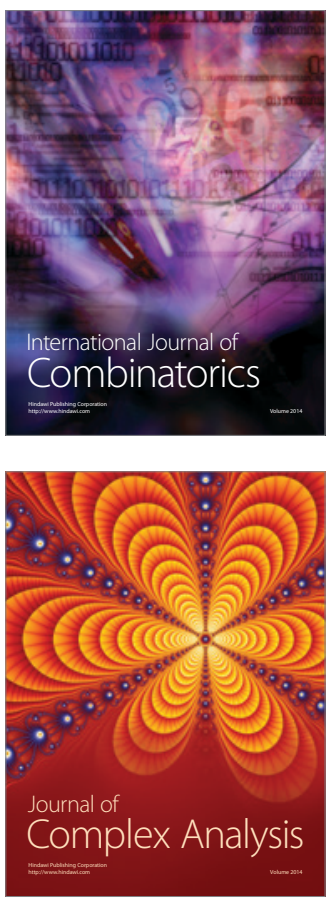

International Journal of

Mathematics and

Mathematical

Sciences
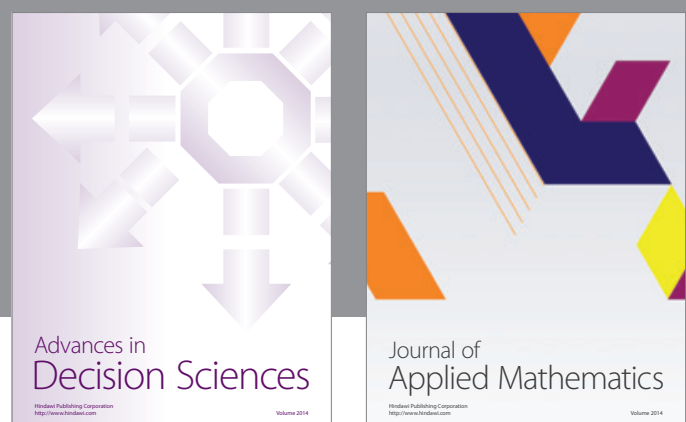

Journal of

Applied Mathematics
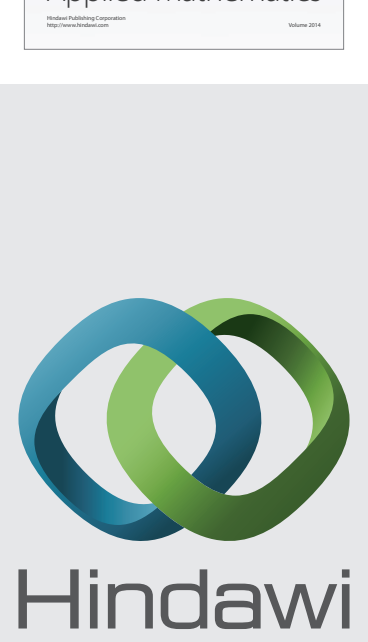

Submit your manuscripts at http://www.hindawi.com
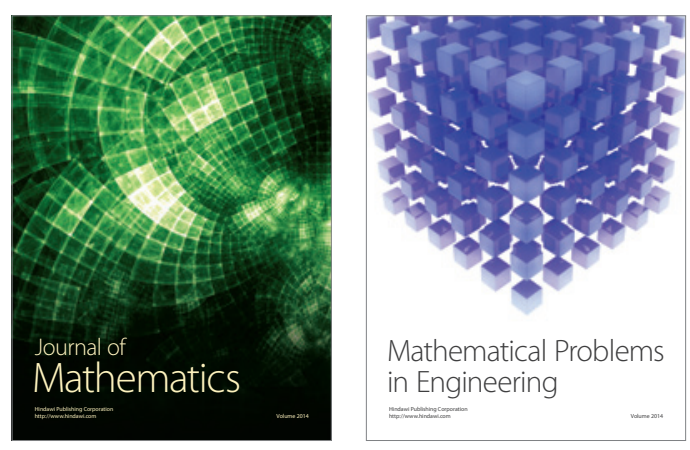

Mathematical Problems in Engineering
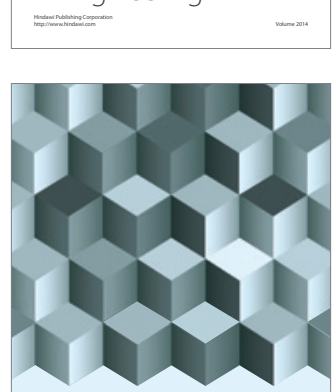

Journal of

Function Spaces
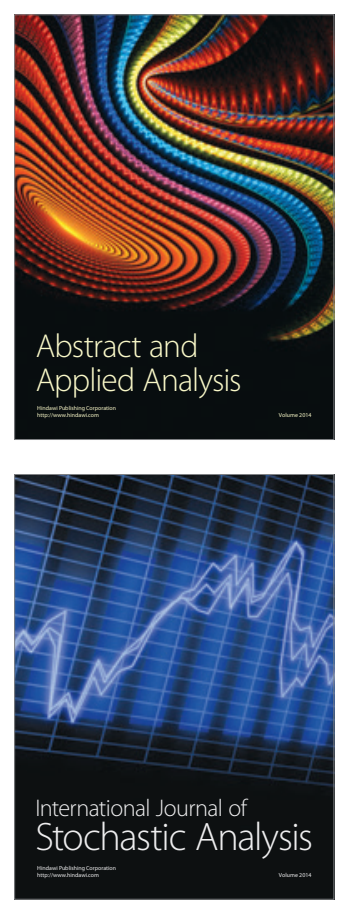

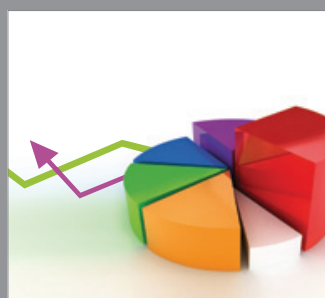

ournal of

Probability and Statistics

Promensencen
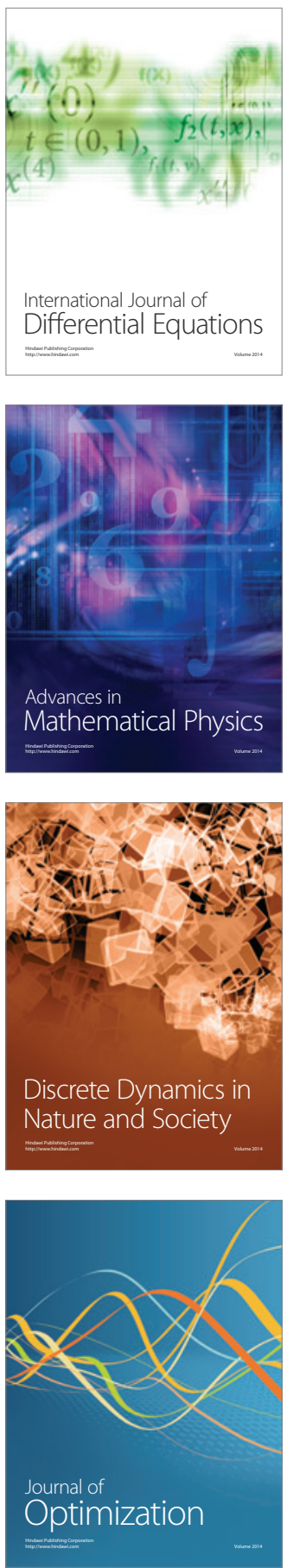